\title{
High Resolution Sculpting and Imaging for Graphene Nano-structures
}

\author{
Wei L. Wang*, David C. Bell** and Efthimios Kaxiras* \\ *Department of Physics and School of Engineering and Applied Sciences \\ **School of Engineering and Applied Sciences and the Center for Nanoscale Systems \\ Harvard University, Cambridge, MA 02138
}

Graphene [1] is a single layer of carbon atoms organized in a honeycomb network which remains stable and very strong both on substrate and when suspended in air or vacuum. Because of its high structure integrity and quasi 2-D carbon crystalline nature, graphene has demonstrated various properties that make it a excellent candidate for the next generation electronics. In the past, molecular systems based on graphene such as carbon nanotube and C60 were intensively studied and showed great promise in exploiting quantum confinement effects and miniaturization of electronic device. However, the drawbacks that limit the application of these systems are the difficulties in sorting, manipulating and integration of them into a circuit. Graphene on the other hand provides a unique opportunity to access the power of the molecular devices through high resolution top-down lithography which can cut an entire circuit of design out a single sheet of graphene.

Since the discovery of graphene, a few attempts have been made to fabricate nanometer scale graphene using lithography based on e-beam [2], scan tunneling microscopy [3] and transmission electron microscopy [4]. These works show that the graphene devices remain stable when approaching to molecular scale and that the resulting devices start showing interesting propeties due to quantum confinement. On the other hand, theoretical works have predicted various interesting electronic and magnetic properties of nanoscale graphene structures such as topological frustration induced magnetism in arbitrary graphene nanoflakes [5] and the halfmetalcity of graphene nanoribbon [6].

Our study combines the power of high resolution transmission electron microscopy / lithography with theoretical first-principles calculation, pushing the fabrication and understanding of graphene sculpted device to the molecular scale. Our state of the art Libra Cs corrected TEM and STEM allows us to punch holes and draw circuits with the nanometer resolution on single, double or few layer graphene. These thin films are suspended across structures or contact electrodes predefined using photolithography. The transport properties can be measured either in situ or out side of TEM vacuum with an option of cyro-cooling. The imaging, configuration and properties of the sculpted molecular devices are simulated with contrast transfer function algorithm, density functional theory and non-equilibrium Green's functional theory. These molecular devices could open doors to many important applications of graphene such as molecular logic processing and single bio-molecular transport and characterization.

References

[1] A. K. Geim and K. S. Novoselov, Nature Materials 6, 183 - 191 (2007)

[2] L. A. Ponomarenko et al., Science 320, 356 (2008).

[3] L. Tapaszto et al., Nat. Nanotechnol. 3, 397 (2008)

[4] M. D. Fischbein and M. Drndic, Applied Physics Lett. 93, 113107 (2008)

[5] W. L. Wang, S. Meng, and E. Kaxiras, Nano Lett. 8, 241(2008). 
[6] Y.-W. Son, M. L. Cohen, and S. G. Louie, Nature (Lon-don) 444, 347 (2006).

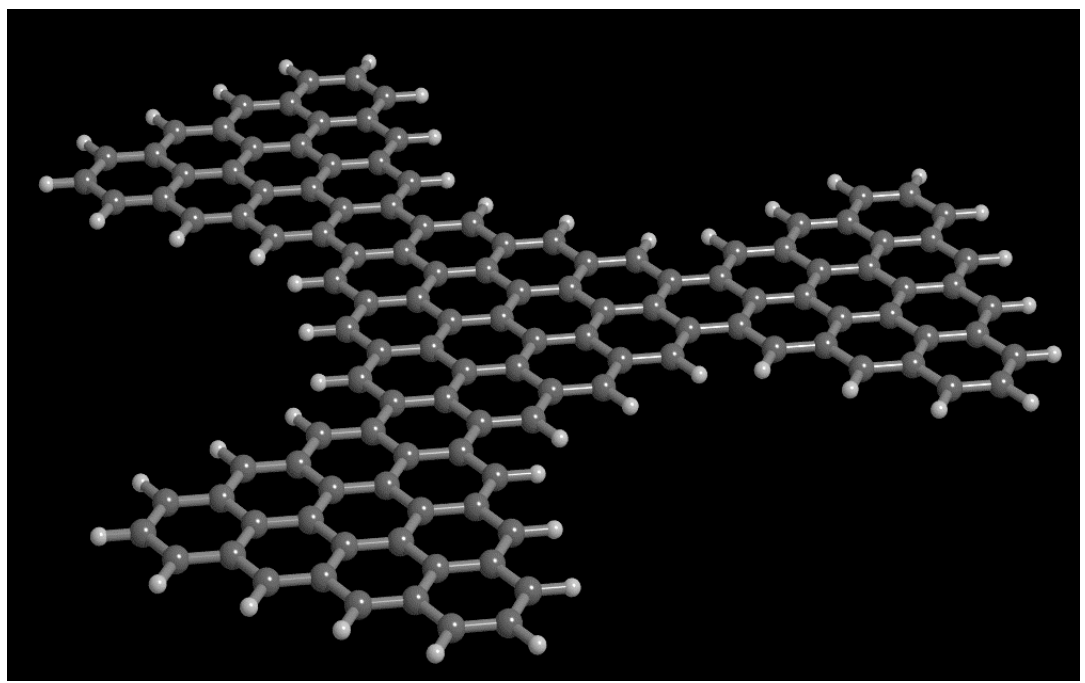

Figure 1. Rendered model of a proposed molecular graphene logic device
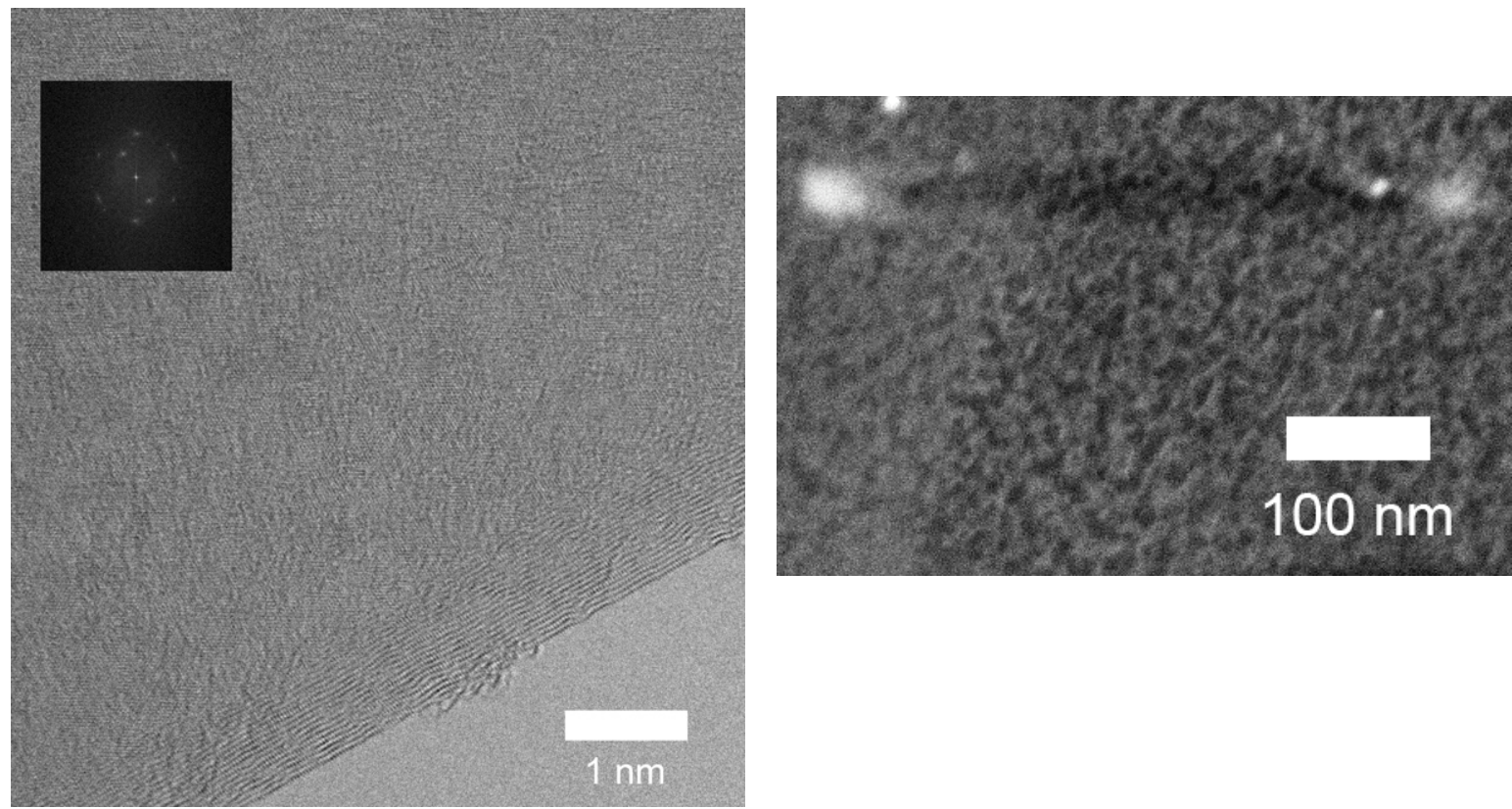

Figure 2 left, HRTEM image of graphene sheet showing folded edge layers and inset FFT form the hexagonal lattice. Right, HAADF image of test cutting of multi-graphene layers using Zeiss Libra 200 STEM showing cutting edges and end dwell points. 